\title{
Refractive status of primary, middle and senior high school students in Shanghai
}

Wang $\mathrm{X}^{1}$, Dong $\mathrm{J}^{2}$, Wu $\mathrm{Q}^{3^{*}}$

${ }^{1}$ Shanxi Eye Hospital, Shanxi, P.R. China.

${ }^{2}$ The First Hospital of Shanxi Medical University, Shanxi, P.R. China.

${ }^{3}$ Affiliated Sixth People's Hospital Shanghai Jiao Tong University, Shanghai, P.R. China.

\section{Abstract}

Aim: To investigate the refractive status in Chinese students ages 7 through 18 years old and to study influencing factors, such as age and gender.

Methods: A total of 748 students (748 eyes) from the primary school group (PS: 483 eyes), middle school group (MS: 78 eyes) and senior high school group (SHS: 187 eyes) were included in this prospective observational case series study. The refractive error values were determined using an automatic refractometer. The spherical refractive error, cylinder power and axis were measured by non-cycloplegic screening.

Results: For spherical error comparison, significant differences existed between every two groups. The SHS showed more negative spherical values compared with the PS $(\mathrm{p}<0.001)$ and MS ( $<0.001)$. The myopia prevalence of MS $(97.4 \%)$ was significantly higher compared with the PS $(70.0 \%)$ and SHS $(80.2 \%)$. The high myopia prevalence of SHS $(19.3 \%)$ was significantly higher compared with the PS $(0.6 \%)$ and MS (2.6\%). Age was negatively correlated with the spherical error (r $=-0.666, \mathrm{p}<0.001)$. Nevertheless, gender had no correlation with it.

Conclusions: With age and studying level increasing, Shanghai urban Chinese students ages 7 through 18 years old showed increasing tendencies towards myopia and high myopia. Thus, closer attention should be paid on the refractive status of this population.

Keywords: Spherical Error; Myopia; High Myopia; Cylinder Power.

\section{Introduction}

With increasing time on reading and doing homework and reducing time on indoor and outdoor activities, the refractive status of students before college plays an important role in the developing visual system. Myopia, as an important cause of correctable visual impairment, showed a high prevalence worldwide, particularly in East Asia [1]. Due to the many potential complications of myopia and high myopia, including myopic retinopathy, myopic macular degeneration, myopic optic neuropathy and retinal detachment, previous studies have investigated the development of myopic shift in East Asia [2-4]. Moreover, population-based studies in Sydney and Singapore showed that older age, type of school, time of outdoor activity and female gender, among other factors, affect myopia development [5-7]. Shanghai, as a commercial and financial center of mainland China, is more intensely competitive and different from the Chinese population who live outside of
Mainland China and Beijing. Thus, we performed this prospective study on school children of urban regions in Shanghai to investigate the refractive status in Chinese students ages 7 through 18 years old and to study influencing factors, such as age and gender.

\section{Methods}

This study was performed according to the guidelines of the Declaration of Helsinki. The subjects were selected from two good teaching quality schools (Rixin Primary School and Nanyang Middle and Senior High School) in Xietu Community of Xuhui District in Shanghai. Only Han-Chinese subjects were selected using the unique ethnicity information on the volunteers' identity cards. This information helped to eliminate any potential influences from different ethnic groups. Ethical approval was obtained from the Shanghai Jiaotong University School of Medicine Ethics Review Board and the Ethics committee of Shanghai Sixth People's

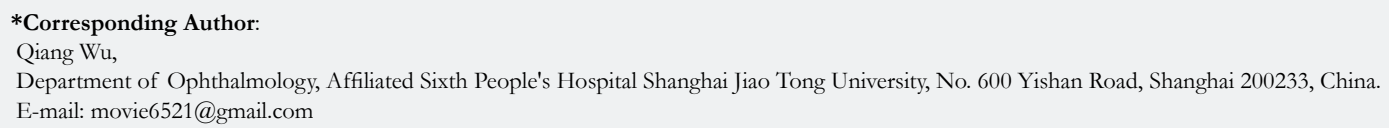

Copyright: Qiang $\mathbf{W u} \mathbf{u}^{\circ}$ 2016. This is an open-access article distributed under the terms of the Creative Commons Attribution License, which permits unrestricted use, distribution and reproduction in any medium, provided the original author and source are credited. 
Hospital. Written informed consent was obtained from each subject's legal guardian after they were provided with an explanation of the nature of the study.

All of the included subjects from the two schools were registered by name, gender and student ID number. Next, a simple ocular examination was performed using a slit-lamp to exclude opacity of the optic media. An automatic refractometer (Auto Refractometer, RM-8800; Topcon Ltd., Tokyo, Japan) examination was performed to obtain a measurement of the refractive status without cycloplegia. The average value of five repetitions was recorded for the final analysis. Based on the level of education, all of the subjects were categorized into the primary school group (PS), middle school group (MS) and senior high school group (SHS).

Statistical analyses were performed using commercial software (SPSS ver. 13.0; SPSS Inc., Chicago, IL, USA). The final results were reported on one random eye of each of the subjects, as the two eyes were significantly correlated for all refractive error values (Pearson correlation coefficient: Spherical error $r=0.904$, $\mathrm{P}<0.001$; Cylinder power $\mathrm{r}=0.729, \mathrm{P}<0.001$; Axis $\mathrm{r}=-0.135$, $\mathrm{P}<0.001)$. All of the numerical data are shown as the mean \pm SD. Myopia was defined as a spherical equivalent refractive error $<-0.5$ diopters (D) and high myopia was defined as a spherical equivalent refractive error that was more negative than -6.0 D [8]. Cylindrical refractions were demonstrated as two perpendicular components in rectangular coordinates for comparison between every two groups [9]. Two-tailed independent sample t-tests were used to compare the mean refractive error values of each group. A general linear model and Pearson correlation analysis were performed to evaluate the relationships between age, gender and refractive status. The Chi-Square test was performed to compare the myopia and high myopia percentile differences between groups. All of the tests had a significance level of $5 \%$.

\section{Results}

A total of 748 subjects (748 eyes), which included 390 male subjects $(52.1 \%)$ and 358 female subjects $(47.9 \%)$, were evaluated using an automatic refractometer. For the entire study population, the mean age was $11.3 \pm 3.36$ years (range: $7-18$ years). The mean spherical error values in PS, MS and SHS were $-0.87 \pm 1.57 \mathrm{D}$, $-3.11 \pm 1.36 \mathrm{D}$ and $-4.20 \pm 2.04 \mathrm{D}$, respectively. The mean spheri- cal error in the SHS was significantly more negative than the PS and MS groups ( $p<0.001$ for both) and a significant difference existed between the PS and MS groups $(p<0.001)$. There were no significant differences in the cylindrical refraction among the three groups using vector analysis. There was no significant difference between males and females in spherical error (Male: -1.88 $\pm 2.31 \mathrm{D}$; Female: $-2.00 \pm 2.14 \mathrm{D} ; \mathrm{p}=0.458)$.

For the PS group, $70.0 \%(338 / 483)$ of the subjects exhibited myopia and $0.6 \%$ (3/483) demonstrated high myopia; for MS, $97.4 \%$ $(76 / 78)$ of the subjects were myopic and $2.6 \%(2 / 78)$ were highly myopic; for SHS, $80.2 \%(150 / 187)$ of the subjects were myopic and $19.3 \%(36 / 187)$ were highly myopic. Pearson Chi-Square test showed that the prevalence of myopia was significantly different between every two groups (PS: MS p<0.001; PS: SHS p $=0.008$; MS: SHS $\mathrm{p}<0.001)$. The high myopia prevalence of SHS was significantly higher than PS and MS ( $p<0.001$ for both), while there was no significant difference between the PS and MS groups $(p=0.144)$.

Pearson correlation analysis showed that the spherical error was negatively correlated with age $(r=-0.666, p<0.001$; Figure $1 \mathrm{~A})$ but not gender $(\mathrm{r}=-0.026, \mathrm{p}=0.485)$. Moreover, the cylindrical refraction was negatively correlated with age $(\mathrm{r}=-0.228, \mathrm{p}<$ 0.001; Figure 1B) but not gender $(\mathrm{r}=0.037, \mathrm{p}=0.312)$. Spherical error showed a positive correlation with cylinder power $(\mathrm{r}=$ $0.188, \mathrm{p}<0.001)$.

\section{Discussion}

As the first study on myopia prevalence in schoolchildren in an urban region of Shanghai, this study demonstrated that with an increase in age and studying level, Chinese school children ages 7 through 18 years old showed increasing myopia and high myopia prevalence.

Our study confirmed some aspects of previous studies on young Chinese populations from Beijing, Singapore and Sydney, such that older age and a higher intensity in studying were independently correlated with a higher prevalence of myopia and high myopia in school children [5-7, 10]. With an increase in studying level from PS to MS and finally to SHS, the sphere error showed

Figure 1 . There was a significant linear correlation between spherical error and age $(\mathrm{A}: \mathrm{r}=-\mathbf{0 . 6 6 6 ,} \mathrm{P}<0.0001)$. The best-fit line $(y=3.053-0.422 x)$ is designated by the solid line; there is a significant linear correlation between cylinder power and age (B: $r=-0.228, P<0.0001)$. The best-fit line $(y=-0.056-0.054 x)$ is designated by the solid line.
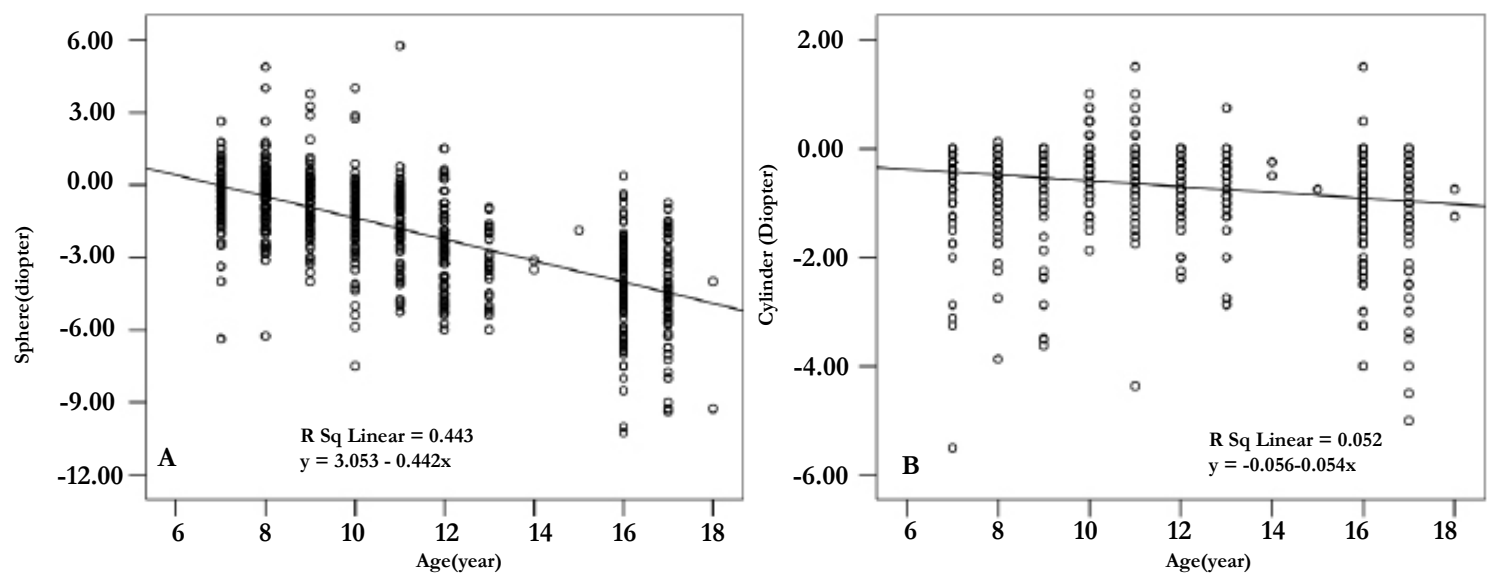
a significant increase, but the prevalence of myopia demonstrated little fluctuation, which may be due to the myopic shift caused by changing coulometric parameters or indoors and outdoors activities $[3,11-13]$. Researchers believed that the education plays an important role in the myopia development $[1-3,5,8,11]$. Compared with the relatively low percentage of high myopia in PS and MS, the significantly increased tendency towards high myopia prevalence in SHS may be attribute to the longer time spent in close reading distance and continuous reading as well as less time spent on outdoor activities [14].

A slightly more negative sphere error in females was observed compared with males in this study, but no significant correlation was found between gender and refractive error, which was consistent with the findings obtained by Ostadimoghaddam et al., who found a lack of significant correlation between gender and myopia based on cycloplegic and non-cycloplegic refraction, which differed from the statistically higher prevalence in females compared with males in Beijing, Singapore and Sydney [5-7, 10, 15]. This difference may be attributed to a different definition for the cut-off values for myopia and cycloplegic or non-cycloplegic refraction.

The refractometer provided mixed astigmatism on the entire ocular system. Previous studies have demonstrated that age showed related changes in the anterior, posterior and total corneal astigmatism, which is consistent with the age-related changes observed in the cylinder power in this study $[16,17]$.

Astigmatism vector, which is represented by the cylinder power and axis, is derived from asymmetries in the anterior ocular segment. Myopia, which is represented by the negative spherical error, results from a longer axial length or cornea/ crystalline lens with too much curvature. The positive correlation between the spherical error and cylinder power may confirm an association between the development of school children myopia and astigmatism in studies by Gwiazda et al [18].

There are several limitations associated with this study. First, cycloplegic refractive measurements were not performed, which may result in a myopic overestimation and hyperopic underestimation due to accommodation [19, 20]. Thus, the prevalence of myopia and high myopia in this study population should be reduced due to the lack of cycloplegia. Second, the sample size of MS and SHS was relatively small and not representative of the population as a whole. For this reason, some findings cannot be simply generalized based on this study alone. Third, the assessing factors associated with myopia in this research was not comprehensive enough. Much more comprehensive influencing factors, such as parental refractive status, reading illumination level, daily studying duration, daily watching television or smart phone duration, studying pressure etc, should be studied. Thus, further studies with cycloplegic measurements, larger sample size and a more comprehensive influencing factors are needed.

\section{Conclusion}

In conclusion, with an increase in age and studying level, Shanghai urban Chinese students ages 7 to 18 years old showed increasing myopia and high myopia prevalence. Thus, closer attention should be paid on the refractive status of this population.

\section{Acknowledgements}

This work is partially supported by the National Natural Science Foundation of China under Grant No. 81501544.

\section{References}

[1]. Pan CW, Ramamurthy D, Saw SM (2012) Worldwide prevalence and risk factors for myopia. Ophthalmic Physiol Opt 32(1): 3-16.

[2]. Wu PC, Tsai CL, Wu HL, Yang YH, Kuo HK (2013) Outdoor activity during class recess reduces myopia onset and progression in school children. Ophthalmology 120(5): 1080-1085.

[3]. Guo Y, Liu LJ, Xu L, Tang P, Lv YY, et al. (2013) Myopic shift and outdoor activity among primary school children: one-year follow-up study in Beijing. PLoS One 8(9): e75260.

[4]. Morgan IG, Ohno-Matsui K, Saw SM (2012) Myopia. Lancet 379(9827): 1739-1748.

[5]. Rose KA, Morgan IG, Smith W, Burlutsky G, Mitchell P, et al. (2008) Myopia, lifestyle, and schooling in students of Chinese ethnicity in Singapore and Sydney. Arch Ophthalmol 126(4): 527-530.

[6]. Saw SM, Carkeet A, Chia KS, Stone RA, Tan DT (2002) Component dependent risk factors for ocular parameters in Singapore Chinese children. Ophthalmology 109(11): 2065-2071.

[7]. Low W, Dirani M, Gazzard G, Chan YH, Zhou HJ, et al. (2010) Family history, near work, outdoor activity, and myopia in Singapore Chinese preschool children. Br J Ophthalmol 94(8): 1012-1016.

[8]. Sun J, Zhou J, Zhao P, Lian J, Zhu H, et al. (2012) High prevalence of myopia and high myopia in 5060 Chinese university students in Shanghai. Invest Ophthalmol Vis Sci 53(12): 7504-7509.

[9]. Krall EM, Arlt EM, Hohensinn M, Moussa S, Jell G, et al. (2015) Vector analysis of astigmatism correction after toric intraocular lens implantation. J Cataract Refract Surg 41(4): 790-799.

[10]. You QS, Wu LJ, Duan JL, Luo YX, Liu LJ, et al. (2012) Factors associated with myopia in school children in China: the Beijing childhood eye study. PLoS One 7(12): e52668.

[11]. Lin Z, Vasudevan B, Jhanji V, Mao GY, Gao TY, et al. (2014) Near work, outdoor activity, and their association with refractive error. Optom Vis Sci 91(4): 376-382.

[12]. Sherwin JC, Reacher MH, Keogh RH, Khawaja AP, Mackey DA, et al. (2012) The association between time spent outdoors and myopia in children and adolescents: a systematic review and meta-analysis. Ophthalmology 119(10): 2141-2151

[13]. Rose KA, Morgan IG, Ip J, Kifley A, Huynh S, et al. (2008) Outdoor activity reduces the prevalence of myopia in children. Ophthalmology 115(8): 1279-1285.

[14]. Ip JM, Saw SM, Rose KA, Morgan IG, Kifley A, et al. (2008) Role of near work in myopia: findings in a sample of Australian school children. Invest Ophthalmol Vis Sci 49(7): 2903-2910.

[15]. Ostadimoghaddam H, Fotouhi A, Hashemi H,Yekta A, Heravian J, et al. (2011) Prevalence of the refractive errors by age and gender: the Mashhad eye study of Iran. Clin Experiment Ophthalmol 39(8): 743-751.

[16]. Ho JD, Liou SW, Tsai RJ, Tsai CY (2010) Effects of aging on anterior and posterior corneal astigmatism. Cornea 29(6): 632-637.

[17]. Ueno Y, Hiraoka T, Beheregaray S, Miyazaki M, Ito M, et al. (2014) Agerelated changes in anterior, posterior, and total corneal astigmatism. J Refract Surg 30(3): 192-197.

[18]. Gwiazda J, Grice K, Held R, McLellan J, Thorn F (2000) Astigmatism and the development of myopia in children. Vision Res 40(8): 1019-1026.

[19]. Fotouhi A, Morgan IG, Iribarren R, Khabazkhoob M, Hashemi H (2012) Validity of noncycloplegic refraction in the assessment of refractive errors: the Tehran Eye Study. Acta Ophthalmol 90(4): 380-386.

[20]. Whatham A, Zimmermann F, Martinez A, Delgado S, de la Jara PL, et al. (2009) Influence of accommodation on off-axis refractive errors in myopic eyes. J Vis 9(3): 14. 1-13.

Special Issue on
"Refractive errors and Strabismus"
Edited by:
Guoqiang Li, The Ohio State University, USA.
E-mail: Guoqiang.Li@osumc.edu

